\title{
REPRESENTATIONS OF ALTERNATIVE CLIFFORD ALGEBRAS OF QUADRATIC FORMS
}

\author{
STACY MARIE MUSGRAVE \\ School of Mathematical and Statistical Sciences, Arizona State University, Tempe AZ 85287, USA
}

(Received 6 October 2013; revised 23 March 2014; accepted 12 May 2014; first published online 18 December 2014)

\begin{abstract}
This work defines a new algebraic structure, to be called an alternative Clifford algebra associated to a given quadratic form. I explored its representations, particularly concentrating on connections to the well-understood octonion algebras. I finished by suggesting directions for future research.
\end{abstract}

2010 Mathematical Subject Classifications. 15A55, 11E88, 17D99

1. Introduction. The purpose of this document is to introduce and explore a new algebraic object, which I will call the alternative Clifford algebra of a quadratic form. This new mathematical object is a generalization of the (associative) Clifford algebra, which was introduced as an algebraic tool to investigate quadratic forms.

Quadratic forms have a rich history in mathematics with many connections to quadratic number fields, geometry, K-theory and various other areas.

In order to study quadratic forms in depth from an algebraic perspective, a structure known as the Clifford algebra was introduced. Clifford algebras are described by a vector space and a quadratic form. Clifford algebras can be described as the 'freest' algebra generated by a given vector space satisfying the condition $v^{2}=q(v) \cdot 1$ for all $v$ in the vector space and a given quadratic form $q$. By studying these algebraic structures, one may draw conclusions about a given quadratic form and vice versa.

It is my purpose in this document to generalize the theory of Clifford algebras by constructing a non-associative algebra in a similar fashion generate the algebra using a vector space in which all vectors satisfy the relation $v^{2}=q(v) \cdot 1$. The motivation behind generating such a structure is to develop a tool to explore quadratic forms using the theory of non-associative algebras. In order to preserve some structure to work with, I require the newly constructed algebra to satisfy the alternative laws.

Of particular relevance is the class of algebras constructed using the CayleyDickson process. This iterative process generates algebras, which I refer to as Cayley algebras, which double in size at each step. To begin the process, take an algebra $A$ of dimension $n$ over $F$ with an involution $a \rightarrow \bar{a}$ satisfying $a+\bar{a} \in F$ and $a \bar{a} \in F$ for all $a \in A$. Choose $\beta \neq 0$ in $F$ and define a new algebra $B=A \oplus A$ with addition and multiplication by scalars defined component-wise, and product given by

$$
(x, y)(w, z)=(x w+\beta z \bar{y}, \bar{x} z+w y) .
$$

Then $B$ is an algebra of dimension $2 n$ over $F$ containing $A$ as a subalgebra. Notice that $B$ also has an involution. Repeat this process on $B$ to obtain a new algebra $C=B \oplus B$ of double the size and also having an involution. This process can 
be repeated infinitely many times, but the properties of the original algebra are quickly lost through iterations of this process. A more detailed description of the Cayley-Dickson process can be found in [3]. This paper will specialize several results to the 8-dimensional octonions produced at the third stage of the Cayley-Dickson process, as their structure and representations are well-understood.

My hope is for this work to lay the foundation for future exploration into the alternative Clifford algebra, as will be discussed in the future work section.

\section{Alternative clifford algebras.}

2.1. Definition of clifford algebra. In the discussion below, let $F$ be any field with char $F \neq 2$. When we say " $F$-algebra", we mean a unital algebra over $F$ which is not necessarily associative.

As mentioned in the introduction, Clifford algebras serve as an algebraic means to study quadratic forms. Loosely speaking, a Clifford algebra is the 'freest' associative algebra generated by a vector space $V$ satisfying $q(v)=v^{2}$ for $v \in V$. A definition in terms of a universal property is given below.

Definition 2.1. An $F$-algebra $A$ is compatible with $q$ if there is an $F$-linear map $\phi: V \rightarrow A$ such that $\phi(x)^{2}=q(x) \cdot 1 \in A$ for any $x \in A$.

We recall two facts for the following lemma. First, every quadratic form $q$ over $V=F^{n}$ has an associated bilinear form $B: V \times V \rightarrow F$, where

$$
B(x, y)=\frac{1}{2}(q(x+y)-q(x)-q(y)) \quad \forall x, y \in V
$$

and

$$
q(x)=B(x, x) \quad \forall x \in V
$$

Second, we have the following definition of a regular quadratic space.

Definition 2.2. A quadratic space $(V, q)$ is regular if either of the following equivalent conditions are true:

- For the bilinear form B corresponding to $\mathrm{q}, x \mapsto B(, x)$ defines an isomorphism $V \rightarrow V^{*}$, where $V^{*}$ denotes the vector space dual of $V$. Recall the vector space dual $V^{*}$ is defined as the set of all linear maps $f: V \rightarrow F$.

- For $x \in V, B(x, y)=0$ for all $y \in V$ implies that $x=0$.

We will concern ourselves only with regular quadratic spaces for the remainder of this paper.

LEMMA 2.3. Let A be compatible with $q$ so there is an associated linear map $\phi: V \rightarrow$ $A$ such that $\phi(x)^{2}=q(x) \cdot 1$ for all $x \in A$. Then $\phi$ is injective, and hence is an embedding of $V$ into $A$. 
Proof. We prove our claim by contrapositive. Suppose $v \neq 0$. Then there is a nonzero $w \in V$ such that $2 B(v, w) \neq 0$ since $q$ is regular. But then

$$
\begin{aligned}
& 0 \neq 2 B(v, w) \\
& 0 \neq q(v+w)-q(v)-q(w)(\text { by definition of B) } \\
& \left.0 \neq \phi(v+w)^{2}-\phi(v)^{2}-\phi(w)^{2} \text { (by definition of } \phi\right) \\
& 0 \neq \phi(v) \phi(w)+\phi(w) \phi(v)(\text { applying linearity of } \phi) .
\end{aligned}
$$

Since $\phi(w) \neq 0$, we conclude $\phi(v) \neq 0$, proving $\phi$ is injective.

Definition 2.4. An associative $F$-algebra $C$ compatible with $q$ is said to be the Clifford algebra for $(V, q)$ if it has the following universal property: given any associative $F$-algebra $A$ compatible with $q$, there exists a unique $F$-algebra homomorphism $\phi$ : $C \rightarrow A$, such that $\phi(x)=x$ for any $x \in V$. We will denote the Clifford algebra for $(V, q)$ by $C l(q)$.

REMARK 2.5. Observe that the algebraic structure of the Clifford algebra is comparable to the quadratic structure of $(V, q)$. In particular, we show below that $x, y \in V$ are orthogonal if and only if $x, y$ anticommute in $A$ (meaning, $x y=-y x$ ). If we let $B$ denote the bilinear form on $V$ associated with $q$, then

$$
2 B(x, y)=q(x+y)-q(x)-q(y)=(x+y)^{2}-x^{2}-y^{2}=x y+y x
$$

So $x$ and $y$ are orthogonal if and only if $x y+y x=0$.

2.2. Definitions and basics of alternative clifford algebra. We now define a new algebra.

Recall the left and right alternative laws:

$$
\begin{array}{ll}
x(x y)=(x x) y & \forall x, y \\
y x(x)=y(x x) & \forall x, y .
\end{array}
$$

The alternative laws imply the flexible law and are equivalent to the Moufang identities, so we also have the latter identities to describe the algebra.

Definition 2.6. An alternative $F$-algebra $C$ compatible with $q$ is said to be the alternative Clifford algebra for $(V, q)$ if it has the following universal property: given any alternative $F$-algebra $A$ compatible with $q$, there exists a unique $F$-algebra homomorphism $\phi: C \rightarrow A$, such that $\phi(x)=x$ for any $x \in V$. We will denote the alternative Clifford algebra for $(V, q)$ by $C l^{A l t}(q)$.

We now look into the generators and relations of the alternative Clifford algebra. In particular, let $\alpha_{1}, \ldots, \alpha_{n}$ generate the basis of the vector space associated to $q$. We consider $C l^{A l t}\left(\left\langle a_{1}, \ldots, a_{n}\right\rangle\right)$ by beginning with the imposed condition that, for $x, y, z \in F$ :

$$
q\left(x_{1} \alpha_{1}+\cdots+x_{n} \alpha_{n}\right)=\left(x_{1} \alpha_{1}+\cdots+x_{n} \alpha_{n}\right)^{2} .
$$

Evaluating the left side gives

$$
a_{1} x_{1}^{2}+\cdots+a_{n} x_{n}^{2}=\left(x_{1} \alpha_{1}+\cdots+x_{n} \alpha_{n}\right)^{2} .
$$


Since this condition must hold for any element in our algebra, we choose specific substitutions to see the relations on generators. In particular, setting $x_{i}=1, x_{j}=0$ for $j \neq i \Rightarrow \alpha_{i}^{2}=a_{i}$ and setting $x_{i}=x_{j}=1, x_{k}=0 \quad$ for $k \neq i, j \Rightarrow \alpha_{i} \alpha_{j}=-\alpha_{j} \alpha_{i}$.

It is clear that given generators $\alpha, \beta, \gamma$ satisfying the above relations that we obtain an algebra $A$ in which $q(v)=v^{2}$ for all $v \in A$.

In order to see what distinguishes this algebra from the standard Clifford algebra $C l(q)$, I specialize to the case of a 3-dimensional form $q=\langle a, b, c\rangle$ with basis elements $\alpha, \beta, \gamma$.

EXAMPLE 2.7. The standard Clifford algebra, $\operatorname{Cl}(\langle a, b, c\rangle)$, consists of the elements $1, \alpha, \beta, \gamma, \alpha \beta, \alpha \gamma, \beta \alpha, \alpha \beta \gamma$. The last element can be written sans parentheses because the standard Clifford algebra is associative, whereas the alternative Clifford algebra has two distinct elements $(\alpha \beta) \gamma$ and $\alpha(\beta \gamma)$ in place of the unparenthesized element in the standard Clifford algebra.

One may wonder if there could be a relation between the elements listed above that allows the alternative Clifford algebra to collapse to 8-dimensions. In order to address this, I must cite future results (Corollary 2.16 and Proposition 2.17). I will show there exist surjective maps $\phi: C l^{A l t}(\langle a, b, c\rangle) \rightarrow O(a, b, c)$ and $\rho: C l^{A l t}(\langle a, b, c\rangle) \rightarrow$ $C l(\langle a, b, c\rangle)$. The latter map implies that

$$
\left|C l^{A l t}(\langle a, b, c\rangle)\right| \geq|C l(\langle a, b, c\rangle)|=8 .
$$

If $\rho$ were an isomorphism (meaning $\left|C l^{A l t}(\langle a, b, c\rangle)\right|=8$ ), then there would be an isomorphism, namely $\phi \circ \rho^{-1}: C l(\langle a, b, c\rangle) \rightarrow O(a, b, c)$, from the associative Clifford algebra $C l(\langle a, b, c\rangle)$ to the non-associative octonion algebra $O(a, b, c)$. This clearly cannot happen, so $\rho$ is not an isomorphism, meaning $\left|C l^{A l t}(\langle a, b, c\rangle)\right|>8$. So the alternative Clifford algebra has at least nine independent elements and is distinct from the standard Clifford algebra.

A natural question to follow that example is:

QuEstion 2.8. What is the dimension of $C l^{A l t}(q)$ ?

This question remains open. It is a nontrivial task to determine whether or not the alternative Clifford algebra is finite-dimensional. As one approach to tackling this question, I explore the representations of the alternative Clifford algebra to the octonions. I will, however, present more general results related to the flexible Clifford algebra (defined in an analogous fashion to the alternative Clifford algebra) and Cayley algebras. At the end of this paper, I will introduce yet another structure to give a sense of what types of results we can hope for with regards to the alternative Clifford algebra.

2.3. Representations. Since the newly defined alternative Clifford algebra shares the common property of being non-associative and satisfying the alternative laws with the octonions, we explore what information we can gather by looking at maps from the alternative Clifford algebra to the octonions. Whenever possible, I generalize to the case of the flexible Clifford algebra and general Cayley algebras, which are flexible. Recall the flexible law states $(x y) x=x(y x) \quad \forall x, y$.

Definition 2.9. A flexible $F$-algebra $C$ compatible with $q$ is said to be the flexible Clifford algebra for $(V, q)$ if it has the following universal property: given any flexible $F$-algebra $A$ compatible with $q$, there exists a unique $F$-algebra homomorphism $\phi$ : 
$C \rightarrow A$, such that $\phi(x)=x$ for any $x \in V$. We will denote the flexible Clifford algebra for $(V, q)$ by $C l^{\text {Flex }}(q)$.

REMARK 2.10. Every Cayley algebra is flexible [3].

First, we recall the fact that the elements which anticommute in a Cayley algebra correspond to those elements which are orthogonal with respect to the norm form $N$. I remind the reader of a few facts.

(1) The norm form $N$ of a Cayley algebra $A$ is an $n$-fold Pfister form. An $n$-fold Pfister form is a quadratic form of the type $\left\langle 1,-a_{1}\right\rangle \otimes \cdots \otimes\left\langle 1,-a_{n}\right\rangle$, for any set of elements $a_{1}, \ldots, a_{n} \in \dot{F}$. We use the notational convention

$$
\left\langle\left\langle a_{1}, \ldots, a_{n}\right\rangle\right\rangle \cong\left\langle 1,-a_{1}\right\rangle \otimes \cdots \otimes\left\langle 1,-a_{n}\right\rangle .
$$

(2) We define the pure part $\phi^{\prime}$ of an $n$-fold Pfister form $\phi=\langle 1\rangle \perp \phi^{\prime}$ to be the $\left(2^{n}-1\right)$ dimensional subform $\phi^{\prime}$.

(3) We refer to the subspace of a Cayley algebra $A$ corresponding to the pure part of the norm form as pure $A$.

Lemma 2.11. Suppose $A$ is a Cayley algebra with norm form $N$. Let $u, v \in$ pure $A$, then $u v=-v u$ if and only if $u \perp v$ with respect to $N$. forms:

Proof. Let $u, v \in$ pure $A$, so $t(u)=t(v)=0$. Note the following facts about norm

(1) The bilinear form $B_{N}(\cdot, \cdot)$ associated to the norm form $N$ can be expressed as $2 B_{N}(x, y)=N(x+y)-N(x)-N(y)$.

(2) All elements in a Cayley algebra satisfy the equation $x^{2}-t(x) x+N(x)=0$, so in particular $N(x)=-x^{2}+t(x) x$ for any $x \in A$.

Now observe that $t(u)=t(v)=t(u+v)=0$. So applying the above equations and simplifying, we get:

$$
\begin{aligned}
2 B_{N}(u, v) & =N(u+v)-N(u)-N(v) \\
& =\left[-(u+v)^{2}+t(u+v)(u+v)\right]-\left[-u^{2}+t(u) u\right]-\left[-v^{2}+t(v) v\right] \\
& =-u^{2}-u v-v u-v^{2}+t(u+v)(u+v)+u^{2}-t(u) u+v^{2}-t(v) v \\
& =-u v-v u
\end{aligned}
$$

So $B_{N}(u, v)=0$ if and only if $u v=-v u$.

We apply this lemma to conclude that most of the time, the image of $(V, q)$ under any map from a flexible Clifford algebra of a quadratic form $q$ is contained in the subspace of the pure part of the Cayley algebra, i.e. the subspace associated to the pure part of the norm form.

Theorem 2.12. Let $A$ be a Cayley algebra with norm form $N$. Given a map $\phi$ : $\mathrm{Cl}^{\text {Flex }}(q) \rightarrow A$, either

(1) $\phi(V) \subset$ pure $A$, or

(2) $q$ is a 1-dimensional form and $\phi(V) \subset F$.

Proof. Let $v \in V$. Because $v$ is an element of the flexible Clifford algebra

$$
v^{2}=q(v) \Rightarrow v^{2}-q(v)=0
$$


Considering the image under the $F$-map $\phi$ yields that $\phi(v)$ satisfies

$$
x^{2}-q(v)=0
$$

Now, $\phi(v) \in A$, so it also satisfies the equation

$$
x^{2}-t(\phi(v)) x+N(\phi(v))=0
$$

Subtracting these equations yields $\phi(v)$ satisfies

$$
t(\phi(v)) x-(q(v)+N(\phi(v))=0 .
$$

So we may consider two cases:

(1) $t(\phi(v))=0$, in which case we are done because $t(\phi(v))=0 \Rightarrow \phi(v) \in$ pure $A$ as desired. Notice that $t(\phi(v))=0$ also implies that $q(v)=-N(\phi(v))$, which we will see in the next lemma.

(2) $t(\phi(v)) \neq 0$, in which case by plugging in $\phi(v)$ into the latter equation, we see that

$$
\phi(v)=[t(\phi(v))]^{-1}(q(v)+N(\phi(v))) \in F \quad(*) .
$$

Now there are subcases to consider here.

(a) If $q(v)=0$, then $v^{2}=q(v)=0$. So $\phi(v)$ satisfies $x^{2}=0$. But then the minimal polynomial for $\phi(v)$ is either

(i) $x=0$, meaning $\phi(v)=0 \Rightarrow t(\phi(v))=0$ and we're done, or

(ii) $x^{2}=0$ and so $[\phi(v)]^{2}=0$. But recall that $(*)$ shows $\phi(v) \in F$ and since $F$ is a domain, there are no zero divisors, so $\phi(v)=0$ and we're back to the previous case.

(b) If $q(v) \neq 0$ and $\phi(v) \neq 0$, then we find an exceptional case as follows. Let $W=$ $v^{\perp}=\left\{w \in V \mid B_{q}(w, v)=0\right\} \subseteq C l^{A l t}(q)$ and let $w \in W$. In $C l^{A l t}(q), w \in W$ means $v w=-w v$ by Lemma 2.11. So under the mapping $\phi$,

$$
\phi(v) \phi(w)=-\phi(w) \phi(v) .
$$

But recall that $\varphi(v) \in F$ by $(*)$, so in particular, $\varphi(v)$ is in the centre and hence commutes with everything, namely

$$
\phi(v) \phi(w)=\phi(w) \phi(v) .
$$

Adding these two equations yields $2 \phi(v) \phi(w)=0$. Again, since $F$ is a domain, there are no zero divisors and so $\phi(v) \neq 0 \Rightarrow \phi(w)=0$. Now we know that $w^{2}-q(w)=0$ because $w \in C l^{A l t}(q)$. So $\phi\left(w^{2}-q(w)\right)=0 \Rightarrow$ $\phi(w)^{2}-q(w)=0$. Since $\phi(w)=0$, we conclude that $q(w)=0$. Now recall that $w$ was an arbitrary element of $W$ and $q$ is a regular quadratic form, so $W=0$. This means that the original vector space is spanned by $v$ and $q$ must be a 1-dimensional quadratic form. Moreover, $(*)$ guarantees that $\phi(V) \subseteq F$.

Next, we establish a general result that if there is a map from the flexible Clifford algebra of $q$ to a Cayley algebra $A$, then the induced map on the vector spaces is actually a quadratic space map. 
Lemma 2.13. Suppose $\operatorname{dim} q \geq 2$ and let $A$ be a Cayley algebra with norm form $N$. If there exists a map $\phi: C l^{\text {Flex }}(q) \rightarrow A$, then the induced map on the associated vector spaces $\tilde{\phi}:(V, q) \rightarrow$ (pure $\left.A,-N^{\prime}\right)$ is a quadratic space map.

Proof. As we saw in the previous theorem, $\phi(V) \subset$ pure $A$. Now the pure part of the Cayley algebra corresponds to the quadratic space associated to the pure part of the norm form $N$, which is a Pfister form and, a priori, a quadratic form. Let us denote the pure part of this Pfister form by $N^{\prime}$.

Now consider

$$
\begin{aligned}
N^{\prime}(\tilde{\phi}(v)) & =-[\tilde{\phi}(v)]^{2}+t(\tilde{\phi}(v)) \\
& =-\tilde{\phi}\left(v^{2}\right)+0 \text { (because } \tilde{\phi}(v) \in \text { pure } A \text { and is a homomorphism) } \\
& \left.=-\tilde{\phi}(q(v)) \text { (because } q(v)=v^{2}\right) \\
& =-q(v) \text { (because } q(v) \in F) .
\end{aligned}
$$

So $\tilde{\phi}$ maps the underlying vector space associated to $\mathrm{Cl}^{\text {Flex }}(q)$ to the underlying vector space associated to pure $A$ and sends $q \rightarrow-N^{\prime}$, showing that $\tilde{\phi}$ is, in fact, a vector space map.

Finally, we establish a result below showing how we have a representation of the flexible Clifford algebra to any algebra constructed with the Cayley-Dickson process.

THEOREM 2.14 (Main representation theorem). Suppose $\operatorname{dim} q \geq 2$ and let $A$ be an algebra constructed using the Cayley-Dickson process through n-iterations. There exists a map from the flexible Clifford algebra

$$
C l^{\text {Flex }}(q) \rightarrow A
$$

if and only if $q \cong-\phi$ where $\phi$ is a subform form of the pure part of the norm form $N$ on the algebra A. Moreover, there is a bijection between $\operatorname{Hom}_{F-a l g}\left(C l^{F l e x}(V, q), A\right)$ and $\operatorname{Hom}_{\text {q.s.maps }}\left((V, q),\left(\{x \in A \mid \operatorname{tr}(x)=0\},-N_{A}^{\prime}\right)\right)$.

Proof. We will show how to construct a quadratic space map given an $F$-algebra map. Suppose there exists a map $\phi: C l^{\text {Flex }}(q) \rightarrow A$. Then consider the restriction of $\phi,\left.\phi\right|_{V}:(V, q) \rightarrow(A, N)$. This is a map of vector spaces $V \rightarrow A$. Moreover, for $v \in$ $V, \phi(v)$ satisfies the characteristic equation $C_{\phi(v)}(x)=x^{2}-t(\phi(v)) x+N(\phi(v))=0$. Namely,

$$
\phi(v)^{2}-t(\phi(v)) \phi(v)+N(\phi(v))=0 .
$$

Since $\operatorname{Im}(V, q) \subset\left(\{\right.$ pure $\left.A\}, N^{\prime}\right), t(\phi(v))=0$ and $N(\phi(v))=N^{\prime}(\phi(v))$, so we get

$$
N(\phi(v))=N^{\prime}(\phi(v))=-\phi(v)^{2} .
$$

Notice that the map from $\mathrm{Cl}^{A l t}(q) \rightarrow A$ being a homomorphism tells us that $0=$ $\phi(0)=\phi\left(v^{2}-q(v)\right)=\phi(v)^{2}-q(v)$, so that $-\phi(v)^{2}=-q(v)$. In particular, this shows that $q(v)=-N^{\prime}(\varphi(v))$, so that $q$ is similar to the pure part of the norm form of $A$. Thus, $\varphi$ is our desired quadratic space map.

Now we show the reverse construction. Suppose we have a quadratic space map $\psi:(V, q) \rightarrow\left(A,-N^{\prime}\right)$ with $q \cong-\phi$ where $\phi$ is a subform form of the pure part of the Pfister form $N=\left\langle\left\langle a_{1}, \ldots, a_{n}\right\rangle\right\rangle$ (denote the pure part by $N^{\prime}$ ). We need only check it 
preserves the relation $q(v)=v^{2}$ to confirm it induces a map on the algebras.

$$
\begin{aligned}
\psi\left(v^{2}-q(v)\right) & =\psi(v)^{2}-\psi(q(v)) \\
& =\psi(v)^{2}-q(v) \text { (because } q(v) \text { is a scalar) } \\
& \left.=\psi(v)^{2}+N^{\prime}(\psi(v)) \text { (because } \psi \text { maps } q \mapsto-N^{\prime}\right) \\
& =\psi(v)^{2}+N(\psi(v)) \text { (because } N=N^{\prime} \text { on the pure part of A) } \\
& =0 .
\end{aligned}
$$

The last line results from the fact that every element of an algebra constructed under the Cayley-Dickson process satisfies $x^{2}-t(x) x+N(x)=0$ and $\psi(v)$ maps to the pure part of the algebra, meaning it has trace 0 . Since $\psi$ preserves the relation, we do in fact obtain a map from $\mathrm{Cl}^{A l t}(q) \rightarrow A$.

This result can then be specialized to the octonion algebra $O(a, b, c)$, showing how the quadratic form we use to construct our alternative Clifford algebra must be related to the norm form of the octonion algebra if a map exists from the former to the latter.

COROLlaRY 2.15. Suppose $\operatorname{dim} q \geq 2$. There exists a map

$$
C l^{A l t}(q) \rightarrow O(a, b, c)
$$

if and only if $q \cong-\phi$ where $\phi$ is a subform form of the pure part of the norm form $\langle\langle a, b, c\rangle\rangle$.

Proof. The octonion algebra $O(a, b, c)$ is a specific case of an algebra constructed with the Cayley-Dickson process through 3 iterations.

The next two results will provide the support for my claims in the Example 2.7. They are followed by a list of results which provide applications of the previous corollary.

COROLlaRY 2.16. There exists a surjective map

$$
C l^{A l t}(\langle a, b, c\rangle) \rightarrow O(a, b, c) .
$$

Proof. The previous corollary states that since $\langle a, b, c\rangle$ is isometric to a scalar multiple of a subform of the pure part of the norm form $\langle\langle a, b, c\rangle\rangle$, there exists a $\operatorname{map} \phi: C^{A l t}(\langle a, b, c\rangle) \rightarrow O(a, b, c)$. Since both algebras are generated by the image of the underlying vector space of the quadratic form, this map must send generators of $C l^{A l t}(\langle\langle a, b, c\rangle\rangle)$ to the generators of $O(a, b, c)$. Hence, it is surjective.

Proposition 2.17. There exists a surjective map

$$
C l^{A l t}(\langle a, b, c\rangle) \rightarrow C l(\langle a, b, c\rangle) .
$$

Proof. The standard Clifford algebra is associative, and hence alternative. The universal property of the alternative Clifford algebra guarantees a unique map from $C l^{A l t}(\langle a, b, c\rangle) \rightarrow C l(\langle a, b, c\rangle)$. As above, this map must send generators to generators. Hence, it is a surjection. 
REMARK 2.18. We can generalize the above proposition to state that there exists a surjective map

$$
C l^{\text {Flex }}\left(\left\langle a_{1}, \ldots, a_{n}\right\rangle\right) \rightarrow A,
$$

where $A$ is a Cayley algebra constructed through $n$ iterations of the Cayley-Dickson process having norm form $\left\langle\left\langle a_{1}, \ldots, a_{n}\right\rangle\right\rangle$. The argument parallels the argument provided in the previous proposition.

Proposition 2.19. Let $q$ be a quadratic form with $\operatorname{dim} q \geq 2$ and Oct be an octonion algebra with norm form $N$. If $1 \in D(q)$ and there is a map $\overline{C l}^{A l t}(q) \rightarrow O c t$, then Oct is split.

Proof. Suppose there is a map $C l^{A l t}(q) \rightarrow$ Oct. Then $-q$ embeds in the pure part of $N$. In particular, since $1 \in D(q),-1 \in D(-q)$ and so $-1 \in D($ pure $N)$. By the Pure Subform Criterion, $N \cong\langle\langle 1, \ldots\rangle$,$\rangle , meaning N$ is hyperbolic and Oct is split.

We return to our study of the alternative Clifford algebra of the 3-dimensional form $q=\langle a, b, c\rangle$.

Lemma 2.20. Let $O\left(a^{\prime}, b^{\prime}, c^{\prime}\right)$ be an octonion algebra and $C^{A l t}(\langle a, b, c\rangle)$ be the alternative Clifford algebra for a quadratic form $q=\langle a, b, c\rangle$. Then if there exists $a$ map

$$
C l^{A l t}(q) \rightarrow O\left(a^{\prime}, b^{\prime}, c^{\prime}\right)
$$

and $q$ is isotropic, then $\left\langle\left\langle a^{\prime}, b^{\prime}, c^{\prime}\right\rangle\right\rangle \in W(F(\langle a, b, c\rangle) / F)$.

Proof. By Corollary 2.1, we know that $q=\langle a, b, c\rangle$ is similar to a subform of the pure part of the Pfister form $\left\langle\left\langle a^{\prime}, b^{\prime}, c^{\prime}\right\rangle\right\rangle$. So if $q$ is isotropic, then so is $\left\langle\left\langle a^{\prime}, b^{\prime}, c^{\prime}\right\rangle\right\rangle$. But as we saw earlier, Pfister forms which are isotropic are also hyperbolic, so $\left\langle\left\langle a^{\prime}, b^{\prime}, c^{\prime}\right\rangle\right\rangle$ is in the Witt kernel $W(F(\langle a, b, c\rangle) / F)$.

This last result allows us to refer Fitzgerald's paper on Witt kernels of quadratic forms [1] to help us determine what restrictions may be placed on $\left\langle\left\langle a^{\prime}, b^{\prime}, c^{\prime}\right\rangle\right\rangle$.

THEOREM 2.21. [Fitzgerald 1.(a)] If $\varphi$ is a neighbour to the $n$-fold Pfister form $\rho$, then $W(F(\phi) / F)$ is a strong $n$-Pfister ideal.

Proposition 2.22. Let $q=\langle a, b, c\rangle$ be a Pfister neighbour to the unique (up to isometry) 2-fold Pfister form $\rho=\langle\langle a b, a c\rangle\rangle$. If there exists a map $C l^{A l t}(q) \rightarrow O\left(a^{\prime}, b^{\prime}, c^{\prime}\right)$, then $\left\langle\left\langle a^{\prime}, b^{\prime}, c^{\prime}\right\rangle\right\rangle \cong\langle\langle a b, a c, t\rangle\rangle$ for some $t \in F$.

Proof. Fitzgerald's result implies that $\left\langle\left\langle a^{\prime}, b^{\prime}, c^{\prime}\right\rangle\right\rangle$ is isometric to a sum of scalar multiples of 2-fold Pfister forms in $W(F(\langle a, b, c\rangle) / F)$. In particular, since the 3fold Pfister form is 8-dimensional, we must express it as the perp of two 2-fold Pfister forms in the Witt kernel. Moreover, since $\langle a, b, c\rangle$ is a Pfister neighbour to $\rho, W(F(\langle a, b, c\rangle) / F)=W(F(\rho) / F)=\rho W F$. Hence, we actually have $\left\langle\left\langle a^{\prime}, b^{\prime}, c^{\prime}\right\rangle\right\rangle=$ $\lambda \rho \perp \gamma \rho=\langle\lambda, \gamma\rangle \rho$ for some $\lambda, \gamma \in F$. 
Now we can see

$$
\begin{aligned}
\left\langle\left\langle a^{\prime}, b^{\prime}, c^{\prime}\right\rangle\right\rangle & =\rho\langle\lambda, \gamma\rangle \\
& =\rho\langle\lambda\rangle\langle\langle\lambda \gamma\rangle\rangle(\text { since }\langle\lambda, \gamma\rangle=\lambda\langle 1, \lambda \gamma\rangle) \\
& =\langle\langle a b, a c, \lambda \gamma\rangle\rangle\langle\lambda\rangle \text { (rewriting } \rho \text { and combining the forms) } \\
& \cong\langle\langle a b, a c, \lambda \gamma\rangle\rangle \text { (see remark below) }
\end{aligned}
$$

So $\left\langle\left\langle a^{\prime}, b^{\prime}, c^{\prime}\right\rangle\right\rangle \cong\langle\langle a b, a c, t\rangle\rangle$ for $t=\lambda \gamma \in F$.

REMARK 2.23. We reference a theorem from Lam (X.1.9) [2] stating that for any Pfister form $\phi$ over $F, D_{F}(\phi)=G_{F}(\phi)$, where $G_{F}(\phi)$ denotes the group of elements $c \in \dot{F}$ satisfying $\langle c\rangle \phi \cong \phi$. In particular, this means that in the above proof, having $1 \in D\left(\left\langle\left\langle a^{\prime}, b^{\prime}, c^{\prime}\right\rangle\right\rangle\right)$ means $1 \in D(\langle\langle a b, a c, \lambda \gamma\rangle\rangle\langle\lambda\rangle)$. And so $\lambda \in D(\langle\langle a b, a c, \lambda \gamma\rangle\rangle)$. By the theorem $D(\langle\langle a b, a c, \lambda \gamma\rangle\rangle)=G(\langle\langle a b, a c, \lambda \gamma\rangle\rangle)$, meaning that $\langle\langle a b, a c, \lambda \gamma\rangle\rangle\langle\lambda\rangle \cong$ $\langle\langle a b, a c, \lambda \gamma\rangle\rangle$.

We generalize the above result using Fitzgerald to get the following.

Proposition 2.24. If $q$ is a neighbour to the $n$-fold Pfister form $\rho$ and there exists a map $C l^{A l t}(q) \rightarrow O\left(a^{\prime}, b^{\prime}, c^{\prime}\right)$, then $\left\langle\left\langle a^{\prime}, b^{\prime}, c^{\prime}\right\rangle\right\rangle \in W(F(q) / F)$, where $W(F(q) / F)$ is a strong $n$-Pfister ideal. This means that $\left\langle\left\langle a^{\prime}, b^{\prime}, c^{\prime}\right\rangle\right\rangle$ is isometric to a sum of scalar multiples of $n$-fold Pfister forms in $W(F(q) / F)$.

REMARK 2.25. Notice that since $\left\langle\left\langle a^{\prime}, b^{\prime}, c^{\prime}\right\rangle\right\rangle$ is only 8-dimensional, the previous corollary only has meaning for $n=2$ or $n=3$ (as there are no octonions to map into for $n \geq 4$ ). We saw the $n=2$ case in Corollary 2.3. The $n=3$ case says that $\left\langle\left\langle a^{\prime}, b^{\prime}, c^{\prime}\right\rangle\right\rangle$ is isometric to a sum of scalar multiples of 3-fold Pfister forms in $W(F(q) / F)$; specifically, $\left\langle\left\langle a^{\prime}, b^{\prime}, c^{\prime}\right\rangle\right\rangle \in W(F(q) / F)$. So for $q$ a Pfister neighbour to $\rho, W(F(q) / F)=W(F(\rho) / F)=\rho W F$, noting that the Witt kernel is a strong Pfister ideal, we see that $\left\langle\left\langle a^{\prime}, b^{\prime}, c^{\prime}\right\rangle\right\rangle \cong \alpha \rho$. In particular, notice that since $1 \in D\left(\left\langle\left\langle a^{\prime}, b^{\prime}, c^{\prime}\right\rangle\right\rangle\right)$ then $1 \in D(\alpha \rho)$. This implies that $\alpha \in D(\rho)$. But we know that $D(\rho)=G(\rho)$ [2], where $G(\rho)$ is the group of similarity scalars. So $\alpha \rho \cong \rho$, and hence $\left\langle\left\langle a^{\prime}, b^{\prime}, c^{\prime}\right\rangle\right\rangle \cong \rho$.

Now, we explore what can be said about the representation when considering an alternative Clifford algebra for a 4-dimensional quadratic form.

COROLlARY 2.26 (Fitzgerald 1.5). Let $\phi$ be an anisotropic form such that $\operatorname{dim} \phi=4$ and $\phi$ is not similar to a Pfister form. If $W(F(\phi) / F) \neq 0$, then $W(F(\phi) / F)$ is a strong 3-Pfister ideal.

COROLlaRY 2.27. Let $q$ be a 4-dimensional anisotropic form. If there exists a map $C l^{A l t}(q) \rightarrow O\left(a^{\prime}, b^{\prime}, c^{\prime}\right)$, then either

$q$ is a conjugate neighbour $\left\langle\left\langle a^{\prime}, b^{\prime}, c^{\prime}\right\rangle\right\rangle \in W(F(q) / F)$ and by the previous corollary, is a strong 3-Pfister ideal.

$q$ is not a conjugate neighbour $O\left(a^{\prime}, b^{\prime}, c^{\prime}\right)$ is trivial $^{1}$.

$q$ is a Pfister form Say $q=\langle\langle x, y\rangle\rangle$, then $\langle\langle a, b, c\rangle\rangle \cong\langle\langle x, y, z\rangle\rangle$ for some $z \in F$.

I employ the fact that Pfister neighbours uniquely determine (up to isometry) the associated Pfister form to make my remark below.

$\overline{{ }^{1} \text { Since }-q} \hookrightarrow 3 \mathbb{\sharp} \perp\langle-1\rangle$ and $\left\langle\left\langle a^{\prime}, b^{\prime}, c^{\prime}\right\rangle\right\rangle \cong 4 \mathbb{U}$ 
Proposition 2.28 (Lam, Proposition X.4.17). Let $\sigma$ be a Pfister neighbour to some $n$-fold Pfister form $\phi$. Suppose $\sigma$ is also a Pfister neighbour to the $n$-fold Pfister form $\psi$. Then $\phi \cong \psi$.

REMARK 2.29. The results for 5-, 6- and 7-dimensional quadratic forms are all the same. For all three cases, if there exists a map $C l^{A l t}(\phi) \rightarrow$ Oct, then $\phi$ embeds in the pure part of the norm form $-N$. In particular, $\phi$ is similar to a subform of $N$, making it a Pfister neighbour by definition. But then $\phi$ uniquely determines $N$. Hence, if a map exists, then $\phi$ is a Pfister neighbour to the norm form $N$ and the octonion algebra $\operatorname{Oct}(N)$ is unique up to isometry.

Note that if $\phi$ is not a Pfister neighbour when $\operatorname{dim} \phi=5,6$ or 7 , then there is no map from the alternative Clifford algebra to an octonion algebra.

3. Further questions. There is a clear need to explore the structure of the alternative Clifford algebra in more depth.

QUESTION 3.1. Is $C l^{A l t}(q)$ finite dimensional?

We would like to make the claim that the alternative Clifford algebra is finite dimensional and, thus, develop a structure theory about alternative Clifford algebras paralleling the existing structure theory of the classic Clifford algebra. In particular, establishing finite-dimensionality would allow one to draw upon the work of Schafer on finite dimensional non-associative algebras [4].

In fact, my original goal was to define a Clifford algebra-esque structure that would satisfy two properties.

(1) It maps into the octonions (so we could compare it to something well-understood).

(2) It satisfies a few specially chosen identities based on properties of the octonions that would result in a finite-dimensional structure.

I originally hypothesized that the alternative laws, or equivalently the Moufang identities, would be sufficient to accomplish these goals. However, there is certainly no restriction forcing us to use the alternative laws to define a new algebra with Clifford algebra qualities. I give an example below of a different direction that could be pursued and list a sample result to give a flavour of what we hope to accomplish in the future with the alternative Clifford algebra. In particular, this new structure can help to address the last comment in Remark 2.6.

QUESTION 3.2. What kind of information can we gather from exploring all the different maps from the alternative Clifford algebra to an octonion algebra? What conditions allow for distinct maps?

Definition 3.3. Let $\Phi=\left\{\phi: C l^{A l t}(q) \rightarrow O \mid O\right.$ is an octonion algebra $\}$ and let $K=$ $\cap_{\phi \in \Phi} \operatorname{ker} \phi$. Define the octonion Clifford algebra $\mathrm{Cl}^{\text {Oct }}(q)$ of a quadratic form $q$ to be the quotient

$$
C l^{A l t}(q) / K
$$

Question 3.4. Is $\mathrm{Cl}^{\mathrm{Oct}}(q)$ finite dimensional?

While I suspect that the answer is yes and further expect it to be easier to show in general than it will be for $\mathrm{Cl}^{A l t}(q)$, I will conservatively state the result for $q$ a Pfister neighbour. 
Proposition 3.5. Let Oct $(\rho)$ be the octonion algebra over $F$ with norm form $\rho$. If $q=$ be the pure part of the Pfister form $\rho$, then $\mathrm{Cl}^{\text {Oct }}(q)$ is finite dimensional.

Proof. Since $q$ is the pure part of the Pfister form $\rho$, by Proposition 2.24 there exists a map $C l^{A l t}(q) \rightarrow O\left(a^{\prime}, b^{\prime}, c^{\prime}\right)$ where $O\left(a^{\prime}, b^{\prime}, c^{\prime}\right) \in W(F(q) / F)$. But, $W(F(q) / F)=$ $W(F(\rho) / F)$, which is generated by $\rho$. So the octonion $O\left(a^{\prime}, b^{\prime}, c^{\prime}\right)$ must be isomorphic to $O(\rho)$. If we consider any two maps $\phi$ and $\phi^{\prime}$ from $C l^{A l t}(q) \rightarrow O(\rho)$, then they must have the same kernel. Namely, if $\phi$ and $\phi^{\prime}$ are induced by the embeddings $\psi: q \rightarrow \rho$ and $\psi^{\prime}: q \rightarrow \rho$. Notice these embeddings can be extended to isomomorphisms $\psi \perp i d: q \perp\langle 1\rangle \rightarrow \rho$ and $\psi^{\prime}$ defined accordingly. But then $(\psi \perp i d)\left(\psi^{\prime} \perp i d\right)^{-1}$ is an isomorphism from $\rho \rightarrow \rho$. This gives us an induced isomorphism on octonions $\alpha: O(\rho) \rightarrow O(\rho)$. In particular, if $x \in \operatorname{ker}(\phi)$, then $\alpha \circ \phi(x)$ sends $x$ to 0 and gives that $x \in \operatorname{ker}\left(\phi^{\prime}\right)$. A similar diagram chase shows that an element of $\operatorname{ker}\left(\phi^{\prime}\right)$ is also in $\operatorname{ker}(\phi)$. So the kernels are the same. Hence, every map $\phi: C l^{A l t}(q) \rightarrow O$ for an octonion $O$ has the same kernel, namely $K=\operatorname{ker} \phi$, giving

$$
C l^{O c t}(q)=C l^{A l t}(q) / K \subset \operatorname{Oct}(\rho) .
$$

Hence $\mathrm{Cl}^{\mathrm{Oct}}(q)$ is finite dimensional because $\operatorname{Oct}(\rho)$ is.

Should one be wary of venturing in this direction and instead insist on concentrating on the alternative Clifford algebra, we could return to the tools presented in this document thus far. In particular, we might try to better understand the representations in hopes of describing the dimension of our new algebra. For instance, one might question:

QUESTION 3.6. Is there a finite number of octonion algebras $O(a, b, c)$ such that there exist maps $\mathrm{Cl}^{\mathrm{Alt}}(q) \rightarrow O(a, b, c)$ ?

I suspect that focusing on the number of distinct images of such a mapping might help us to answer the ultimate question regarding finite-dimensionality.

This points to another point of interest about my results. Many of them rely on the supposition of existence of a representation to then say something of note. We would hope to achieve a more complete theory to include when such a map exists. For example, Corollary 2.3 states that if a map exists $C l^{A l t}(q) \rightarrow O\left(a^{\prime}, b^{\prime}, c^{\prime}\right)$, then $\left\langle\left\langle a^{\prime}, b^{\prime}, c^{\prime}\right\rangle\right\rangle \cong\langle\langle a b, a c, t\rangle\rangle$ for some $t \in F$. It would be highly desirable for this statement to be an 'if and only if' statement, or at least an 'if' and 'only if plus something more'.

It is my hope that by highlighting some of these points, the interested reader might pursue further study of this work.

\section{REFERENCES}

1. R. W. Fitzgerald, Witt kernels of function field extensions. Pac. J. Math. 109 (1), (1983) 89-106.

2. T. Y. Lam, Introduction to quadratic forms over fields, in Graduate Studies in Mathematics vol. 67 (American Mathematical Society, Providence, RI, 2005).

3. R. D. Schafer, On the algebras formed by the Cayley-Dickson process, Am. J. Math. 76 (1954), 435-446.

4. R. D. Schafer, An introduction to nonassociative algebras (Dover Publications Inc., New York, 1995.) Corrected reprint of the 1966 original. 